\title{
Modeling of fluorescence line-narrowed spectra in weakly coupled dimers in the presence of excitation energy transfer
}

\author{
Chen Lin, ${ }^{1, a)}$ Mike Reppert, ${ }^{1, b)}$ Ximao Feng, ${ }^{1, c)}$ and Ryszard Jankowiak ${ }^{1,2, d)}$ \\ ${ }^{1}$ Department of Chemistry, Kansas State University, Manhattan, Kansas 66506, USA \\ ${ }^{2}$ Department of Physics, Kansas State University, Manhattan, Kansas 66506, USA
}

(Received 26 March 2014; accepted 24 June 2014; published online 15 July 2014)

\begin{abstract}
This work describes simple analytical formulas to describe the fluorescence line-narrowed (FLN) spectra of weakly coupled chromophores in the presence of excitation energy transfer (EET). Modeling studies for dimer systems (assuming low fluence and weak coupling) show that the FLN spectra (including absorption and emission spectra) calculated for various dimers using our model are in good agreement with spectra calculated by: (i) the simple convolution method and (ii) the more rigorous treatment using the Redfield approach [T. Renger and R. A. Marcus, J. Chem. Phys. 116, 9997 (2002)]. The calculated FLN spectra in the presence of EET of all three approaches are very similar. We argue that our approach provides a simplified and computationally more efficient description of FLN spectra in the presence of EET. This method also has been applied to FLN spectra obtained for the CP47 antenna complex of Photosystem II reported by Neupane et al. [J. Am. Chem. Soc. 132, 4214 (2010)], which indicated the presence of uncorrelated EET between pigments contributing to the two lowest energy (overlapping) exciton states, each mostly localized on a single chromophore. Calculated and experimental FLN spectra for CP47 complex show very good qualitative agreement. (C) 2014 AIP Publishing LLC. [http://dx.doi.org/10.1063/1.4887083]
\end{abstract}

\section{INTRODUCTION}

Fluorescence line-narrowing spectroscopy (FLNS) is a low-temperature spectroscopic technique which has been successfully employed over the years in detection/ characterization of a wide variety of systems, e.g., organic molecules, ${ }^{1-3}$ inorganic systems, ${ }^{4}$ DNA and protein adducts (Ref. 5 and references therein). This technique also provides considerable insight into the electronic structure and excitation energy-transfer (EET) dynamics of excitonically coupled systems such as photosynthetic complexes. ${ }^{6}$ One characteristic of photosynthetic systems is fast EET; thus FLN spectroscopy can provide information about the nature of low-energy states, excitonic interactions between coupled high- and low-energy pigments, and basic physical parameters of the system, such as electron-phonon (el-ph) coupling strength. Delta FLN $(\triangle \mathrm{FLN})$ spectroscopy, i.e., the difference between two consecutive FLN measurements separated by a certain period of spectral hole-burning (SHB), can also provide information on the spectral density. ${ }^{7,8}$ However, many modeling studies of FLN spectra followed a traditional approach to treat systems as dilute glasses, ${ }^{9-12}$ i.e., completely neglecting the effects of possible energy transfer and/or excitonic delocalization, which may be present in

a) Current address: Department of Chemistry, University of California, Merced, 5200 North Lake Road, Merced, California 95343, USA.

b) Current address: Department of Chemistry and James Frank Institute, The University of Chicago, 929 E 57th Street, Chicago, Illinois 60637, USA.

c) Current address: Alliance Fiber Optic Products, 275 Gibraltar Drive, Sunnyvale, California 94089, USA.

d) Author to whom correspondence should be addressed. Electronic mail: ryszard@ksu.edu complex biological systems (e.g., in various photosynthetic antenna pigment complexes). Only in computational studies based on experimental data $^{13}$ and theoretical modeling by Renger et al. using the Redfield theory-based energy transfer calculations, ${ }^{14}$ these two effects have been taken into account.

In our recent modeling studies,${ }^{13}$ Monte Carlo simulation was used to treat the random disorder of pigments site energies, and each set of site-energies was treated by a Hamiltonian with coupling strength between pigments reflected by the non-diagonal elements. A pure electronic spectrum is an average over disorder in site energies, and transition lineshapes are obtained by simply convoluting pure electronic spectra with single site lineshape function. ${ }^{13}$ In Renger and Marcus' approach $^{14}$ using Redfield theory, ${ }^{15-17}$ a density matrix description of transition lineshapes replaces this simple convolution method (SCM) in order to more accurately reflect the effects of electron-phonon coupling and energy-transfer lifetime broadening on the calculated optical spectra. The importance of lifetime broadening was also illustrated by modeling of resonant hole-burned (HB) spectra for various model dimers. ${ }^{18}$ Though for stronger el-ph coupling strength $(S>1)$, different lineshape theories must be employed, as suggested in Refs. 19-21, the Redfield approach (RA) is an appropriate approximation for systems with low el-ph coupling strength.

However, in order to achieve reasonable signal-to-noise ratio in calculated FLN spectra using the RA, calculations are computationally time-consuming and are generally beyond the capabilities of a simple desktop computer. One possible way to ease the calculation processes of FLN spectra (in the presence of EET) is to develop analytical formulas for weak coupling conditions, including only the ef- 
fects of energy transfer between different chromophores in the complex and ignoring explicit coupling effects. Under weak inter-pigment coupling and weak el-ph coupling conditions, where uncorrelated EET and efficient Förster energy transfer between pigments can take place, Reppert $e t$ al. ${ }^{22}$ developed analytical formulas to describe the process of uncorrelated EET and to model nonphotochemical hole-burned (NPHB) spectra. In this work, the latter model ${ }^{22}$ is extended to simulate resonant FLN spectra in the presence of EET (modeling of $\triangle F L N$ spectra will be reported elsewhere). We show below that the analytical formulas developed to describe FLN spectra provide a very good approximation of both SCM and RA type calculations, assuming that both excitonic and el-ph couplings are weak. That is, the coupling matrix element $\left(\mathrm{V}_{\mathrm{nm}}\right)$ must be smaller than the reorganization energy $\left(\mathrm{E}_{\lambda}\right)$ and $S \leq 1$. We demonstrate that our method provides very smooth spectra with a very short computation time (a few seconds) for a single FLN spectrum. That is, the computation time using our analytical formulas has been decreased by many orders of magnitude in comparison with the RA simulations. This work will also show that our model is applicable to describe the main features of the experimental FLN spectra obtained for the CP47 antenna of Photosystem II (PSII) ${ }^{13}$ in which the lowest-energy $\mathrm{Q}_{\mathrm{y}}$-bands were mostly contributed to by two weakly coupled Chls. ${ }^{13}$ The shapes of FLN spectra clearly suggested the presence of EET within the two low-energy absorption bands.

\section{DERIVATION OF ANALYTICAL FORMULAS TO CALCULATE FLN SPECTRA IN THE PRESENCE OF EET}

For a multi-chromophore system, which consists of $N$ weakly coupled pigments, pigment $i(1 \leq i \leq N)$ has SDF $f(\omega)$ with a Gaussian lineshape centered at $\omega_{i}{ }^{22}$ There are two components for pigment $i$. One component is a trap state $L_{i}(\omega)$, which is already at the lowest energy and is not capable to transfer energy; the other component allows EET to other pigments, and is labeled with $U_{i}(\omega) . L_{i}(\omega)$ and $U_{i}(\omega)$ can be expressed as

$$
\begin{aligned}
L_{i}(\omega) & =P\left(\omega_{i} \approx \omega\right) \cdot \prod_{\substack{j=1 \\
j \neq i}}^{N} P\left(\omega_{j}>\omega\right) \\
& =f_{i}(\omega) \cdot \prod_{\substack{j=1 \\
j \neq i}}^{N}\left(1-\int_{-\infty}^{\omega} f_{j}(t) d t\right),
\end{aligned}
$$

and

$$
\begin{aligned}
U_{i}(\omega) & =f_{i}(\omega)-L_{i}(\omega) \\
& =f_{i}(\omega) \cdot\left(1-\prod_{\substack{j=1 \\
j \neq i}}^{N}\left(1-\int_{-\infty}^{\omega} f_{j}(t) d t\right)\right),
\end{aligned}
$$

where $P\left(\omega_{i} \approx \omega\right)$ stands for the probability for $\omega_{i} \approx \omega$ in pigment $i$ (it equals $\left.f_{i}(\omega)\right)$ and $P\left(\omega_{j}>\omega\right)$ is the probability when $\omega_{j}>\omega$ in pigment $j$ (i.e., $1-\int_{-\infty}^{\omega} f_{j}(t) d t$ ). The joint probability gives the distribution of trap-state.

Single-site spectra for all pigments are $\sigma\left(\omega-\omega_{Z P L}\right)$ in absorption and $\sigma\left(\omega_{Z P L}-\omega\right)$ in fluorescence. The probability that a trap-state molecule of type $i$ having ZPL frequency $\omega$ will be excited resonantly by the laser is given by

$$
F_{R, i}(\omega)=L_{i}(\omega) \cdot \sigma\left(\omega_{e x c}-\omega\right),
$$

where $\omega_{\text {exc }}$ stands for the excitation frequency. Similarly, the probability that a non-trap state molecule of type $i$ will be excited is given by $U_{i}(\omega) \cdot \sigma\left(\omega_{\text {exc }}-\omega\right)$. The fluorescence signal originating from resonant excitation of trap-state pigments is given (as in the absence of energy transfer) as the convolution of the $F_{R}(\omega)$ expression with the single-site fluorescence spectrum.

In the case of the emission spectrum originating from excitation of a non-trap state pigment of type $i$, after such an excitation has occurred, emission will occur not from pigment $i$ but from the lowest energy trap in the complex. The probability density function for trap state pigments of types other than $i$ is given by

$$
\sum_{\substack{j=1 \\ j \neq i}}^{N} L_{j}(\omega)
$$

However, if excitation occurs at some frequency $\omega_{0}$, it is clear that the lowest-energy trap state must have ZPL frequency lower than $\omega_{0}$; so given excitation of a non-trap pigment $i$ at frequency $\omega_{0}$, the distribution function for potential emitting states becomes

$$
\begin{aligned}
& \underset{\left(-\infty, \omega_{o}\right)}{\chi}(\omega) \cdot n_{i} \cdot \sum_{\substack{j=1 \\
j \neq i}}^{N} L_{j}(\omega)=\left\{\begin{array}{cc}
n_{i} \cdot \sum_{\substack{j=1 \\
j \neq i}}^{N} L_{j}(\omega), & \left(\omega \leq \omega_{o}\right) \\
0, & \left(\omega>\omega_{o}\right)
\end{array}\right. \\
& \text { Here } n_{i}=\left(\int_{-\infty}^{\omega_{e x c}}\left[\sum_{\substack{j=1 \\
j \neq i}}^{N} L_{j}(\omega)\right] d \omega\right)^{-1}
\end{aligned}
$$

is a normalization factor to give the entire distribution unit probability. The probability of such an excitation occurring is given by $U_{i}\left(\omega_{o}\right) \cdot \sigma\left(\omega_{e x c}-\omega_{o}\right)$, so that the probability density function for molecules emitting at frequency $\omega$ after absorption by a higher-energy pigment $i$ at frequency $\omega_{0}$ becomes

$$
U_{i}\left(\omega_{o}\right) \cdot \sigma\left(\omega_{\text {exc }}-\omega_{o}\right) \cdot \underset{\left(-\infty, \omega_{o}\right)}{\chi}(\omega) \cdot n_{i} \cdot \sum_{\substack{j=1 \\ j \neq i}}^{N} L_{j}(\omega)
$$

Integrating over all possible excitation frequencies removes the dependence on $\omega_{0}$ : 


$$
\begin{aligned}
\int_{-\infty}^{\infty} U_{i}\left(\omega_{o}\right) \cdot \sigma\left(\omega_{\text {exc }}-\omega_{o}\right) \cdot \underset{\left(-\infty, \omega_{o}\right)}{\chi}(\omega) \cdot n_{i} \cdot \sum_{\substack{j=1 \\
j \neq i}}^{N} L_{j}(\omega) d \omega_{o} \\
=\left[\int_{-\infty}^{\infty} U_{i}\left(\omega_{o}\right) \cdot \sigma\left(\omega_{\text {exc }}-\omega_{o}\right) \cdot \underset{\substack{\left(-\infty, \omega_{o}\right) \\
\chi}}{\chi}(\omega) d \omega_{o}\right] \\
\cdot n_{i} \cdot \sum_{\substack{j=1 \\
j \neq i}}^{N} L_{j}(\omega) .
\end{aligned}
$$

Considering for a moment $\omega$ as a fixed value, the function $\chi(\omega)$ is zero when $\omega>\omega_{o}$ and is equal to 1 when $\omega$ $\left(-\infty, \omega_{o}\right)$

$\leq \omega_{o}$. The integrand $U_{i}\left(\omega_{o}\right) \cdot \sigma\left(\omega_{\text {exc }}-\omega_{o}\right) \cdot \underset{\left(-\infty, \omega_{o}\right)}{\chi}(\omega)$ is thus also zero when $\omega>\omega_{o}$ and is equal to $U_{i}\left(\omega_{o}\right) \cdot \sigma\left(\omega_{\text {exc }}-\omega_{o}\right)$ when $\omega \leq \omega_{o}$. The integral can thus also be expressed as

$$
\left[n_{i} \cdot \int_{\omega}^{\infty} U_{i}\left(\omega_{o}\right) \cdot \sigma\left(\omega_{e x c}-\omega_{o}\right) d \omega_{o}\right] \cdot \sum_{\substack{j=1 \\ j \neq i}}^{N} L_{j}(\omega) .
$$

There will be $N$ such probability density functions, reflecting excitation of the non-trap distributions of each of the $N$ pigments in the complex. The total non-resonant emission spectrum is given by

$$
\begin{aligned}
& F_{N R}(\omega) \\
& =\sum_{i=1}^{N}\left[\left(n_{i} \cdot \int_{\omega}^{\infty} U_{i}\left(\omega_{o}\right) \cdot \sigma\left(\omega_{\text {exc }}-\omega_{o}\right) d \omega_{o}\right) \cdot \sum_{\substack{j=1 \\
j \neq i}}^{N} L_{j}(\omega)\right] \\
& =\sum_{i=1}^{N} \sum_{\substack{j=1 \\
j \neq i}}^{N}\left[L_{j}(\omega) \cdot n_{i} \cdot \int_{\omega}^{\infty} U_{i}\left(\omega_{o}\right) \cdot \sigma\left(\omega_{\text {exc }}-\omega_{o}\right) d \omega_{o}\right] \\
& =\sum_{\substack{i, j=1 \\
i \neq j}}^{N} L_{j}(\omega) \cdot n_{i} \cdot \int_{\omega}^{\infty} U_{i}\left(\omega_{o}\right) \cdot \sigma\left(\omega_{\text {exc }}-\omega_{o}\right) d \omega_{o} .
\end{aligned}
$$

The total fluorescence distribution function is

$$
F(\omega)=F_{N R}(\omega)+F_{R}(\omega) .
$$

The actual fluorescence spectrum can be calculated as the convolution of this function with the single-site fluorescence spectrum $F_{F l u}$ :

$$
\begin{aligned}
\operatorname{FLNS}(\omega)= & {\left[\sum_{i=1}^{N} L_{i}(\omega) \cdot F_{A b s, i}\left(\omega_{e x c}-\omega\right)\right.} \\
& +\sum_{\substack{i, j=1 \\
i \neq j}}^{N} L_{j}(\omega) \cdot n_{i} \cdot \int_{\omega}^{\infty} U_{i}\left(\omega_{o}\right) \\
& \left.\cdot F_{A b s, i}\left(\omega_{e x c}-\omega_{o}\right) d \omega_{o}\right] * F_{F l u} .
\end{aligned}
$$

In this equation, $\mathrm{F}_{A b s, i}\left(\omega_{e x c}-\omega\right)$ is the reflection of singlesite absorption spectrum with respect to excitation frequency for pigment $i$. In this method, assuming all pigments have the same transition dipole moment, bulk absorption spectrum is obtained by convoluting the sum of SDFs for all pigments with single-site absorption, while bulk emission is calculated by convoluting the sum of trap state from all pigments $\sum_{i=1}^{N} L_{i}(\omega)$ with single-site fluorescence.

Though the method developed in this work does not include pigments' coupling constant under the assumption of weak coupling, this parameter is involved in the calculation of simple convolution method and the Redfield approach. Thus, in the model calculations for a weakly coupled dimer, using our analytical formulas, we consider effects of three parameters: (i) site energy differences, (ii) the strength of the el-ph coupling (Huang-Rhys factor $S$ ), and (iii) inter-pigment coupling constant.

\section{RESULTS AND DISCUSSION}

\section{A. Spectral density}

The spectral density $J(\omega)$ provides information about the reservoir and its interaction with the relevant system; it is important to describe bath relaxation dynamics associated with the exciton transfer process. ${ }^{14}$ Coupling to the bath reveals which frequencies are available in the bath to relax. At $T=0 \mathrm{~K}$, the Huang-Rhys factor $(S)$ is given by ${ }^{23}$

$$
S=\int_{0}^{\infty} d \omega J(\omega) .
$$

At the same time, reorganization energy $\left(E_{\lambda}\right)$ is defined as

$$
E_{\lambda}=\hbar \int_{0}^{\infty} \omega J(\omega) d \omega .
$$

This parameter is the energy cost due to geometry modifications. ${ }^{14}$ The profile of spectral density can be obtained by fitting the experimental shape of the phonon sideband in the $\triangle$ FLN spectrum excited in the low-energy wing of the SDF. (We emphasize that the proper shape of $J(\omega)$ cannot be obtained from simple FLN spectra, as often reported in the literature). ${ }^{14,24,25}$ For more details on $\triangle$ FLN spectra and challenges in measuring the Huang-Rhys factor $(S)$ see Refs. 8 and 26.

Various empirical functional forms for $J(\omega)$ are used in simulations, and one frequently used function for spectral density in the HB community is half-Gaussian halfLorentzian (HG-HL) shape (Refs. 6 and 27, and references therein), as its shape is similar to the experimental onephonon profile. This form, however, cannot be used in Redfield calculation as it leads to infinitely large $E_{\lambda}$ (for details see Ref. 7) and cannot be used in energy transfer expressions; therefore, a lognormal distribution function is used in this work to describe the shape of spectral density, ${ }^{7}$ i.e.,

$$
J(\omega)=\frac{S}{\omega \sigma \sqrt{2 \pi}} e^{-\frac{\left[\ln \left(\omega / \omega_{c}\right)\right]^{2}}{2 \sigma^{2}}},
$$

which also provided excellent fits of experimental spectral densities obtained for various photosynthetic complexes. ${ }^{7}$ In Eq. (14), $S$ is the integrated area of spectral density, and equals 
the value of Huang-Rhys factor. Parameters $\sigma$ and $\omega_{c}$ are standard deviation and mean of the lognormal distribution. Substituting Eq. (14) into the integral in Eq. (13) yields a welldefined $E_{\lambda}$.

To calculate $J(\omega)$ we use $\sigma=0.764$ and $\omega_{c}=52.0 \mathrm{~cm}^{-1}$. This spectral density resembles HG-HL shape of the onephonon profile with the mean phonon frequency $\omega_{m}$ of $26 \mathrm{~cm}^{-1}$ and the FWHM $=26 \mathrm{~cm}^{-1}$ (for Gaussian part) and $85 \mathrm{~cm}^{-1}$ (for Lorentzian part) of $J(\omega)$. The parameters used in our modeling studies are reasonable, as similar parameters were observed in many photosynthetic antenna pigment complexes. ${ }^{7,28-30}$

\section{B. Building single-site spectrum for the analytical expressions method}

According to Ref. 6, within the Franck-Condon approximation and low-temperature limit $(T \rightarrow 0 \mathrm{~K})$, single-site absorption (SSA) and single-site emission (SSE) spectra can be expressed as

$$
\begin{aligned}
L_{A / F}(\omega)= & e^{-S} l_{0}\left(\omega-\Omega_{0}\right) \\
& +\sum_{R=1}^{\infty} S^{R} \frac{e^{-S}}{R !} l_{R}\left(\omega-\Omega_{0} \mp R \omega_{m}\right) .
\end{aligned}
$$

In this equation, $L_{A / F}(\omega)$ is single-site spectrum for absorption/emission; $l_{0}\left(\omega-\Omega_{0}\right)$ is the Lorentzian lineshape of the ZPL peaking at $\Omega_{0} ; l_{\mathrm{R}}\left(\omega-\Omega_{0} \pm \mathrm{R} \omega_{\mathrm{m}}\right)$ stands for the normalized line-shape function of the $R$-phonon transition $(R=1,2$, ... ) with peak position at $\Omega_{0} \pm R \omega_{\mathrm{m}}$ ( + sign for emission and - sign for absorption). A Poisson weighting factor for every $R$ determines the intensity distribution of the PSB. The profile $l_{R}$ is obtained by convolution of $l_{1}$ with itself $(R-1)$ times. $l_{1}$ is one-phonon profile and equals the normalized spectral density. Thus, in this work, for absorption, $l_{1}\left(\omega-\Omega_{0}-R \omega_{\mathrm{m}}\right)$ can be described by Eq. (14).

\section{Comparison of three methods to calculate FLN spectra}

As an instructive example, we consider a weakly coupled dimer composed of A- and B-pigments. Thus, Eq. (11) can be rewritten as

$$
\begin{aligned}
& \operatorname{FLNS}(\omega) \\
& \begin{array}{l}
=\left[L_{A}(\omega) \cdot F_{A b s, A}\left(\omega_{\text {exc }}-\omega\right)+L_{B}(\omega) \cdot F_{A b s, B}\left(\omega_{\text {exc }}-\omega\right)\right. \\
\quad+L_{A}(\omega) \cdot n_{B} \cdot \int_{\omega}^{\infty} U_{B}\left(\omega_{o}\right) \cdot F_{A b s, B}\left(\omega_{\text {exc }}-\omega_{o}\right) d \omega_{o} \\
\left.\quad+L_{B}(\omega) \cdot n_{A} \cdot \int_{\omega}^{\infty} U_{A}\left(\omega_{o}\right) \cdot F_{A b s, A}\left(\omega_{\text {exc }}-\omega_{o}\right) d \omega_{o}\right] * F_{F l u} .
\end{array}
\end{aligned}
$$

We assume that both pigments have Gaussian shape of the SDF with a FWHM of $200 \mathrm{~cm}^{-1}$. Several categories of pigment interaction are considered in the calculation, i.e., the electronic coupling strength ranges from extreme weak (coupling constant $\mathrm{V}_{\mathrm{AB}}$ is set to $\left.0 \mathrm{~cm}^{-1}\right)$ to moderately weak $\left(\mathrm{V}_{\mathrm{AB}}\right.$ $=25 \mathrm{~cm}^{-1}$ ). Very small coupling matrix elements (i.e., $\mathrm{V}_{\mathrm{AB}}$ $\sim 0-2 \mathrm{~cm}^{-1}$ ) are observed between very weakly interacting pigments in various antenna pigment complexes. For example, the coupling constant between Chl514 and Chl526 in CP47 antenna system ${ }^{13}$ is close to zero, while the coupling constant between Chl523 and Chl 526 is $\sim 2 \mathrm{~cm}^{-1}$. However, this is not a general case for weakly coupled dimers. Thus, in the calculation, we also investigate situations for other weak coupling constants (i.e., coupling constant is smaller than reorganization energy).

\section{Zero-coupling limit $\left(V_{A B}=0 \mathrm{~cm}^{-1}\right)$.}

Figure 1 shows a degenerate case where the site energies of both pigments (A and B) are set at $15000 \mathrm{~cm}^{-1}$, with the Huang-Rhys factor of 0.2 and coupling constant $\mathrm{V}_{\mathrm{AB}}=$ $0 \mathrm{~cm}^{-1}$. For simplicity, we refer to the calculation method developed in this work as the analytical expression method. Calculated spectra shown in Figures 3 and 4 follow the same format as that in Figure 1, unless otherwise stated.

It is clear that under this parameter set, all three calculation methods provided almost identical spectra obtained for

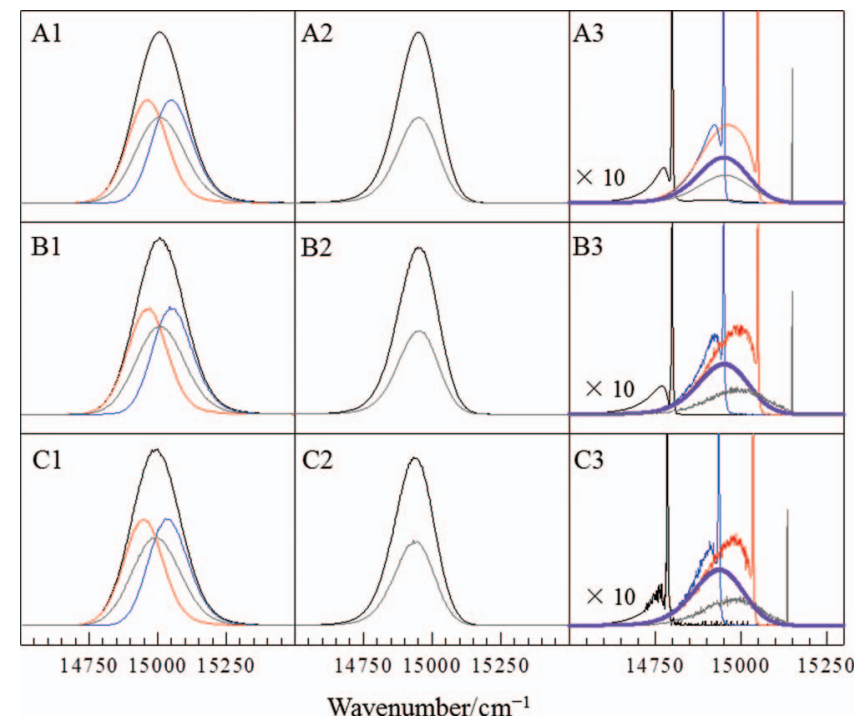

FIG. 1. Calculated spectra of weakly coupled dimer with degenerate site energies at $15000 \mathrm{~cm}^{-1}\left(\mathrm{~V}_{\mathrm{AB}}=0 \mathrm{~cm}^{-1}\right)$; inhomogeneous broadening $\left(\Gamma_{\text {inh }}\right.$ $=200 \mathrm{~cm}^{-1}$; Huang-Rhys factor $S=0.2$; and mean phonon frequency $\omega_{m}$ $=26 \mathrm{~cm}^{-1}$. Capital letters in upper left corner of all frames label rows, and numbers right after capital letters label columns. Rows A, B, and C are calculation results using our analytical expressions, SCM, and the RA, respectively. Columns 1, 2, and 3 correspond to calculated absorption, emission, and FLN spectra, respectively. In column 1, black lines are calculated bulk spectra; red and blue curves stand for contributions from the pigments from trap-state and EET component, respectively; gray lines are contributions from each pigment. Column 2 shows total emission (black) and pigment's contribution (gray). In the third column, black, blue, red, and gray spectra are collected with excitation frequency of $14800 \mathrm{~cm}^{-1}, 14950 \mathrm{~cm}^{-1}, 15050 \mathrm{~cm}^{-1}$, and $15150 \mathrm{~cm}^{-1}$, respectively (note, excitation frequencies are red-shifted by $14 \mathrm{~cm}^{-1}$ in RA methodology to correct the shift due to reorganization energy); thick purple lines are calculated emission spectra (same as black curves in column 2 in each corresponding row). Figures 3-5 have the same organization unless otherwise stated. 
the bulk absorption and emission spectra. The only difference is that the peak positions of the absorption and emission spectra calculated by the RA, as expected, must be corrected (i.e., red-shifted) by the value of reorganization energy, i.e., in this case by about $14 \mathrm{~cm}^{-1}$ when $S=0.2$. Column 3 shows the calculated FLN spectra at various excitation frequencies with three different methods (see figure caption for details). When the excitation frequency is at the low-energy side of the SDF (and $S<1$ ), FLN signal has significantly larger contribution from resonant emission than non-resonant emission, resulting in FLN spectra with very intense zero-phonon lines (ZPLs) and weak phonon side-bands (PSBs); see black curves in column $3\left(\omega_{\text {exc }}=14800 \mathrm{~cm}^{-1}\right)$ of Figure 1. Note that in order to better resolve the shape of PSBs, black spectra are multiplied by the factor showed in each corresponding frame. All three methods (i.e., analytical formulas, SCM, and RA) show the same trend of the change of FLN spectra with different excitation frequencies; that is, the higher the excitation frequency, the more contribution from non-resonant emission (due to a fast EET process from higher energy pigment to the low energy pigment) is observed, and FLN spectra resemble the bulk emission spectra (compare with the purple curves in frames $\mathrm{A} 3, \mathrm{~B} 3$, and $\mathrm{C} 3$ ).

The similarity of FLN spectra calculated by three methods is further investigated by comparing the resonant ( $\mathrm{rFLN}$ ) and non-resonant (nrFLN) contributions to FLN signal (Figure 2 and Table I). Figure 2 qualitatively shows that under a certain excitation frequency, the lineshapes of FLN sig-

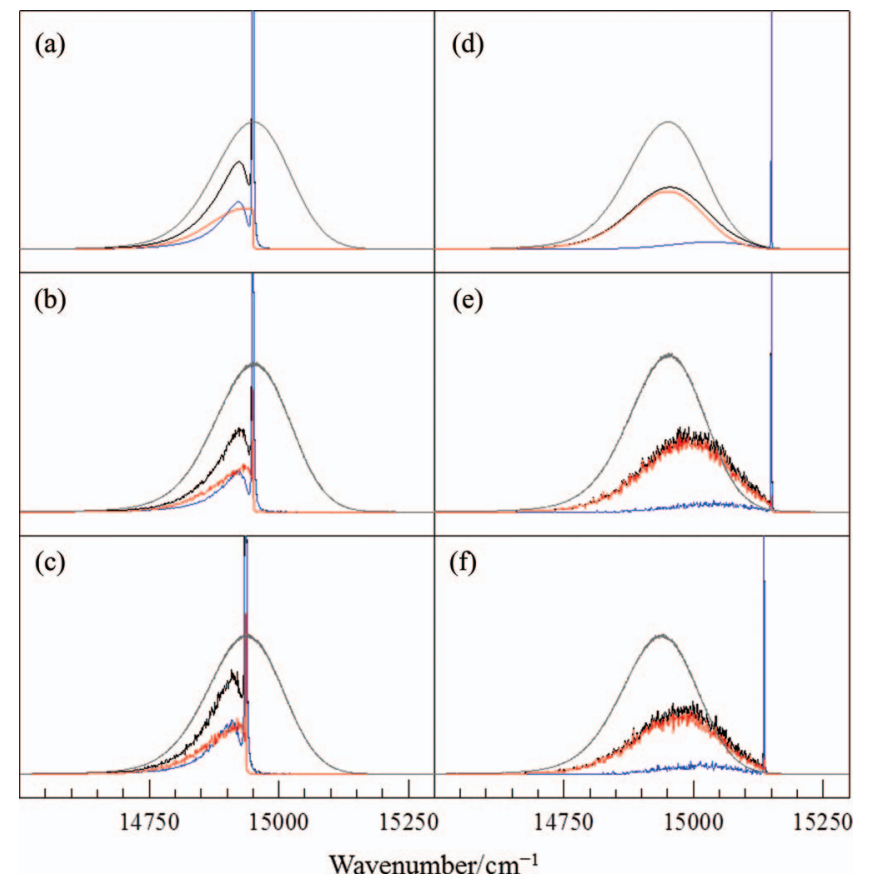

FIG. 2. Calculated spectra of weakly coupled dimer with degenerate site energy at $15000 \mathrm{~cm}^{-1}$. Coupling constant is $0 \mathrm{~cm}^{-1}$; inhomogeneous broadening is $200 \mathrm{~cm}^{-1}$; Huang-Rhys factor $S$ equals to 0.2 ; mean phonon frequency is $26 \mathrm{~cm}^{-1}$. Black, blue, red, and gray curves stand for total FLN, resonant part of FLN, non-resonant contribution of FLN, and total emission, respectively. A: $\omega_{\mathrm{ex}}=14950 \mathrm{~cm}^{-1}$, AE method; B: $\omega_{\mathrm{ex}}=15150 \mathrm{~cm}^{-1}$, AE method; C: $\omega_{\mathrm{ex}}=14950 \mathrm{~cm}^{-1}$, SCM; D: $\omega_{\mathrm{ex}}=15150 \mathrm{~cm}^{-1}, \mathrm{SCM}$; E: $\omega_{\text {ex }}=14936 \mathrm{~cm}^{-1}$, RA; F: $\omega_{\text {ex }}=15136 \mathrm{~cm}^{-1}$, RA. Note in Redfield calculation, excitation frequency is red-shifted by $14 \mathrm{~cm}^{-1}$.
TABLE I. Relative integrated intensity of resonant ( $\mathrm{rFLN}$ ) and non-resonant contributions for FLN spectra (nrFLN) calculated by AE, SCM, and RA (based on Figure 2).

\begin{tabular}{lccc}
\hline \hline $\begin{array}{l}\text { Calculation } \\
\text { method }\end{array}$ & $\begin{array}{l}\text { Analytical } \\
\text { expression }\end{array}$ & $\begin{array}{c}\text { Simple convolution } \\
\text { method }\end{array}$ & $\begin{array}{c}\text { Redfield } \\
\text { approach }\end{array}$ \\
\hline rFLN/FLN $\left(14950 \mathrm{~cm}^{-1}\right)$ & 0.737 & 0.733 & 0.739 \\
$\operatorname{nrFLN} /$ FLN $\left(14950 \mathrm{~cm}^{-1}\right)$ & 0.263 & 0.267 & 0.261 \\
$\mathrm{rFLN} / \mathrm{FLN}\left(15150 \mathrm{~cm}^{-1}\right)$ & 0.124 & 0.104 & 0.122 \\
nrFLN/FLN $\left(15150 \mathrm{~cm}^{-1}\right)$ & 0.876 & 0.896 & 0.878 \\
\hline \hline
\end{tabular}

nals, as well as resonant (blue curves) and non-resonant (red curves) contributions, are very close despite different calculation methods. Table I is a quantitative comparison which shows the integrated intensity ratio of rFLN (or nrFLN) with respect to FLN spectra. It is clear from the table that the FLN spectra calculated by all three methods under the speculated condition are essentially similar.

Figure 3 shows calculated spectra for larger $S$ value $(S$ $=0.8$ ). Note that for $\mathrm{V}_{\mathrm{AB}}=0 \mathrm{~cm}^{-1}$ and $\Gamma_{\mathrm{inh}}=200 \mathrm{~cm}^{-1}$ the absorption and emission maxima, calculated spectra by the RA are (as expected) red-shifted by $\sim 55 \mathrm{~cm}^{-1}$ in comparison with spectra obtained via our analytical expressions and SCM. Therefore, in order to match the relative position of the excitation on the bulk absorption spectrum, the excitation frequencies chosen for FLN calculation in the RA methodology are $55 \mathrm{~cm}^{-1}$ lower in frequency. Recall that a direct comparison of FLN spectra using the three methods described above can be only made as long as the bulk absorption spectra match. Note that the FLN spectra calculated with all three methodologies for larger $S$-value $(S=0.8)$ are also very similar.

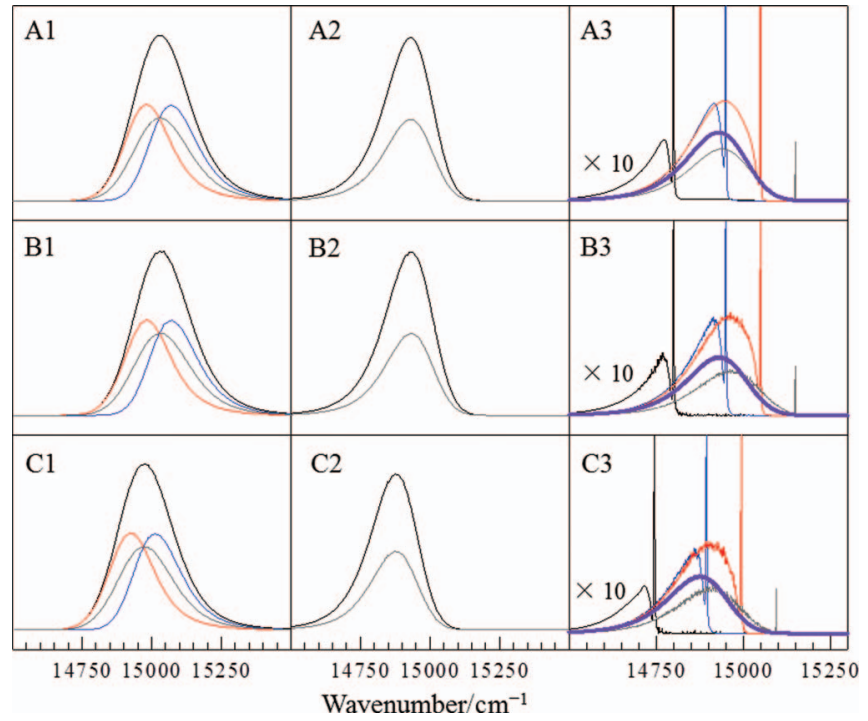

FIG. 3. Calculated spectra of weakly coupled dimer with degenerate site energies at $15000 \mathrm{~cm}^{-1}$. Coupling constant is $\mathrm{V}_{\mathrm{AB}}=0 \mathrm{~cm}^{-1}$; inhomogeneous broadening is $\Gamma_{\text {inh }}=200 \mathrm{~cm}^{-1}$; Huang-Rhys factor $S=0.8$. In the third column, black, blue, red, and gray spectra are collected with excitation wavenumber at $14800 \mathrm{~cm}^{-1}, 14950 \mathrm{~cm}^{-1}, 15050 \mathrm{~cm}^{-1}$, and $15150 \mathrm{~cm}^{-1}$, respectively. Note, excitation frequencies to generate data shown in frame $\mathrm{C} 3$ (RA methodology) are red-shifted by $55 \mathrm{~cm}^{-1}$ to correct for the shift due to reorganization energy. 
The same methodologies discussed above, were also used to perform calculation for the non-degenerate dimers with site energies at $14950 \mathrm{~cm}^{-1}$ and $15050 \mathrm{~cm}^{-1}$ and the coupling constant $\mathrm{V}_{\mathrm{AB}}=0 \mathrm{~cm}^{-1}$ as shown in Figures SI-1 and SI-2 in the supplementary material. ${ }^{41}$ Unlike in the case of degenerate dimers, where both type of pigments contribute equally to trap- and/or energy transfer state, in the case of non-degenerate dimer the trap state is mainly localized on pigments with lower site-energy, while the state which allows EET is mostly localized on pigments with higher site-energy. Therefore, the two pigments have different contribution to the bulk emission spectra. Except for this difference, the same conclusions, as those obtained based on data presented in Figures 1-3, are valid. Thus, we conclude that in the presence of EET, when pigments have extremely weak intermolecular coupling constants, our analytical formula provides an excellent approximation for SCM and the RA calculations of the FLN spectra. Regarding calculation time, our new and simple method only takes several seconds; while in order to get similar smoothness for calculated FLN spectra the SCM and RA simulations require the calculation program to be run on a computer cluster (i.e., Beocat in KSU) for at least a couple of hours. Therefore, the calculating method with analytical expression discussed above is preferred in simulating FLN spectra for dimers with extremely weak coupling constants in the presence of EET.

\section{Moderately weak coupling $\left(V_{A B}=25 \mathrm{~cm}^{-1}\right.$ and $10 \mathrm{~cm}^{-1}$ ).}

Figure 4 shows spectra obtained for $\mathrm{V}_{\mathrm{AB}}=25 \mathrm{~cm}^{-1}$ and $S=0.8$. Note that under this condition, i.e., $\mathrm{V}_{\mathrm{AB}}$ is smaller than reorganization energy $\left(55 \mathrm{~cm}^{-1}\right)$, this case also falls into the regime of weak coupling. However, unlike in frame A1 of

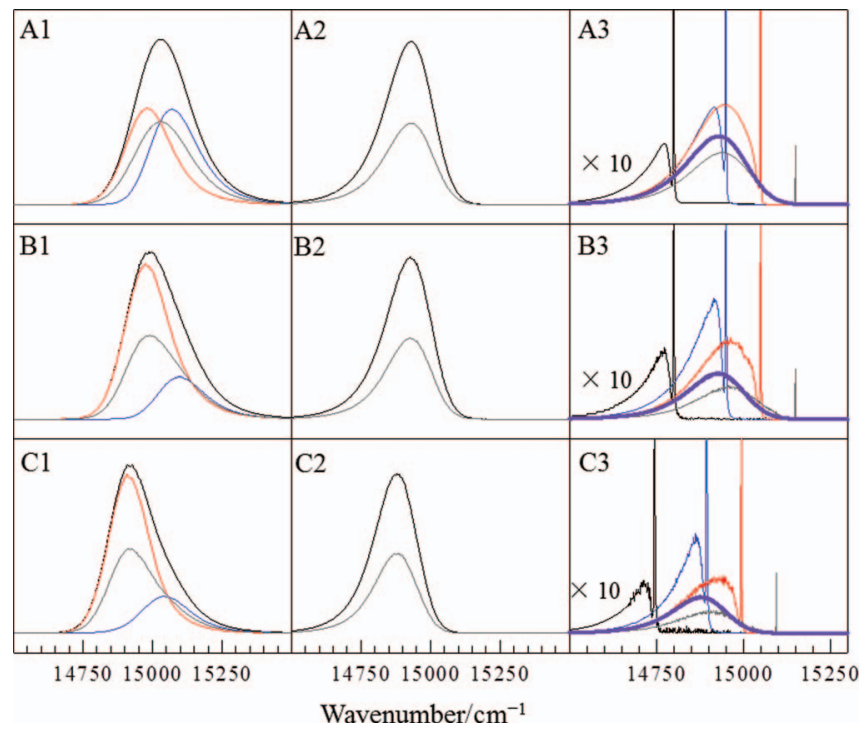

FIG. 4. Calculated spectra of a weakly coupled dimer with degenerate site energies of $15000 \mathrm{~cm}^{-1}$. Coupling constant is $25 \mathrm{~cm}^{-1} ; \Gamma_{\text {inh }}=200 \mathrm{~cm}^{-1}$; $S=0.8$. In the third column, black, blue, red, and gray spectra are collected with excitation wavenumber at $14800,14950,15050$, and $15150 \mathrm{~cm}^{-1}$, respectively (excitation frequencies are red-shifted by $55 \mathrm{~cm}^{-1}$ in RA calculations to correct for the shift due to $\mathrm{E}_{\lambda}$ ).
Figure 4, where upper and lower excitonic states contribute equivalently to the total absorption spectrum (due to the fact that coupling constant is not involved in the calculation by analytical expression method), both SCM and RA-type simulations show larger contribution from lower excitonic state (trap state), resulting in narrower bandwidths of absorption spectra shown in frames B1 and $\mathrm{C} 1$. This is due to the fact that excitonic delocalization is taken into account in both SCM and RA, and the width of the distribution of excitonic energies experiences a narrowing that scales with the square root of the delocalization number in Redfield calculation. ${ }^{31,32}$ Recall that the Redfield theory also takes energy-transfer lifetime into account, thus the higher excitonic state (blue line in frame $\mathrm{C} 1$ of Figure 4) is slightly broader and less intense than that calculated by SCM (blue line in frame B1 of Figure 4). On the contrary, as expected, emission spectra calculated by three methods (black curves in column 2 of Figure 4) do not reveal large changes. That is, emission spectra in frame A2 and B2 are similar, suggesting that for $\mathrm{V}_{\mathrm{AB}}=25 \mathrm{~cm}^{-1}$, our derived formulas are still a good approximation for SCM. Hence, a negligible difference should be observed in the FLN spectra calculated by our derived analytical formulas and SCM simulation, in agreement with our modeling results shown in Figure 4 (frames A3, B3, and C3). In the calculation of FLN spectra we only consider the emission from a trap state, which has a long lifetime compared to the higher-energy (i.e., the EET) state. Therefore, FLN spectra calculated by all three methods have narrow ZPLs. However, lifetime broadening of ZPLs can be accurately modeled by RA. ${ }^{18}$

In the Redfield calculations (RA; row 3 of Figure 4), emission spectrum is narrowed by $\sim 5 \%$ comparing to black curves in frames A2 and B2. This is because the effective $S$ factor involved in RA for a spectral profile is smaller than the input Huang-Rhys factor (apparent $S$ factor). When a smaller apparent $S$ factor is chosen, this narrowing effect becomes negligible. For example, Figure SI-3 in the supplementary material. ${ }^{41}$ shows calculated spectra for $S=0.2$ and $\mathrm{V}_{\mathrm{AB}}$ $=25 \mathrm{~cm}^{-1}$. Though in Figure SI-3 of the supplementary material, ${ }^{41}$ conditions for calculations no longer meet the criteria for a weak coupling limit, which requires coupling constant smaller than $\mathrm{E}_{\lambda}\left(\mathrm{E}_{\lambda}\right.$ is $\sim 14 \mathrm{~cm}^{-1}$ when $\left.S=0.2\right)$, the expected narrowing of the emission spectrum becomes negligibly small. The small amount of narrowing in emission spectra calculated by Redfield theory is also observed in the case of a non-degenerate dimer (when $\mathrm{V}_{\mathrm{AB}}=25 \mathrm{~cm}^{-1}$ ); see Figures SI-4 and SI-5 in the supplementary material. ${ }^{41}$

Figure 5 shows spectra obtained with $\mathrm{V}_{\mathrm{AB}}=10 \mathrm{~cm}^{-1}$. Row A is the same as that shown in Figures 3 and 4. The most obvious difference between methods is observed in the calculated absorption spectra. That is, in the SCM and RA methods a contribution from the upper excitonic state in Figure 5 is relatively larger when compared with data obtained for $\mathrm{V}_{\mathrm{AB}}$ $=25 \mathrm{~cm}^{-1}$ in Figure 4. In summary, our calculations reveal that by increasing the coupling constant to $25 \mathrm{~cm}^{-1}$, the difference between absorption spectra obtained by $\mathrm{AE}$ and SCM/RA methods becomes larger, due to the fact that coupling constants are not explicitly involved in AE-type calculation. To extend our AE approach one could also include absorption lineshape function, wavelength-dependent EET, 


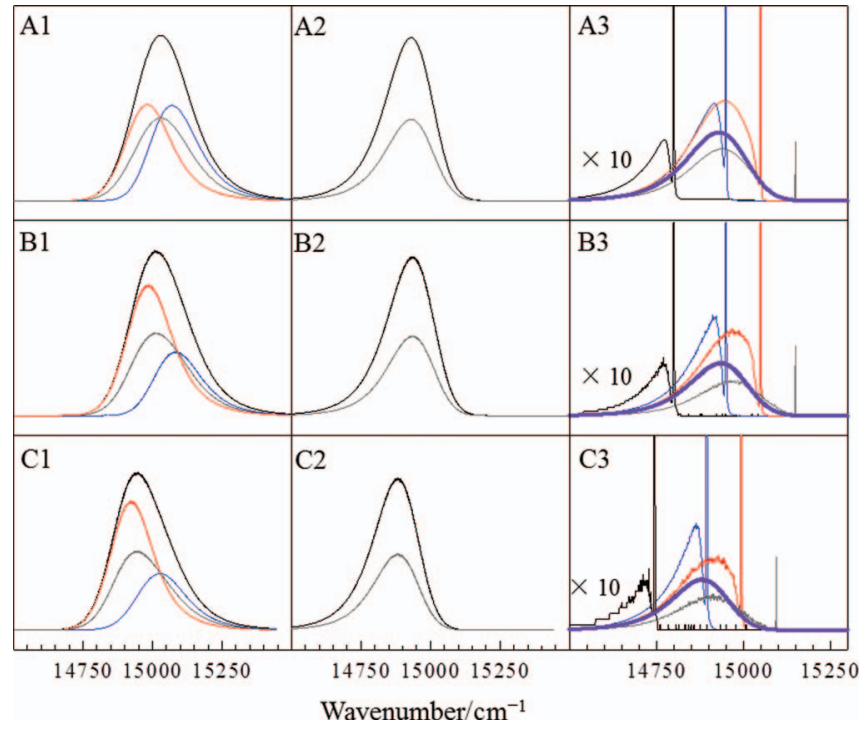

FIG. 5. Calculated spectra of a weakly coupled dimer with degenerate site energies of $15000 \mathrm{~cm}^{-1}$. Coupling constant is $10 \mathrm{~cm}^{-1} ; \Gamma_{\mathrm{inh}}=200 \mathrm{~cm}^{-1}$; $S=0.8$. In the third column, black, blue, red, and gray spectra are collected with excitation frequency of $14800,14950,15050$, and $15150 \mathrm{~cm}^{-1}$, respectively. (Note that excitation frequencies are red-shifted by $55 \mathrm{~cm}^{-1}$ in RA calculations to correct for the shift due to $\mathrm{E}_{\lambda}$.)

and spectral hole-burning following EET. However, the AE model, as described, is a very good approximation to calculate FLN spectra when coupling constants and Huang-Rhys factor are small.

\section{Fluorescence line-narrowed spectra of CP47 antenna complex}

$\mathrm{CP} 47$ is one of the core antenna protein complexes of PSII, which binds 16 Chls. ${ }^{33,34}$ By simulating CP47 absorption, emission, and nonresonant HB spectra, Reppert et al. reported $^{13}$ that the two lowest energy $\mathrm{Q}_{\mathrm{y}}$-absorption bands (partly degenerate) are mostly localized on two weakly coupled pigments, i.e., pigment $\mathrm{A}=\mathrm{Chl} 523$ and pigment $\mathrm{B}=\mathrm{Chl} 526$, with the coupling constant $\mathrm{V}_{\mathrm{AB}}$ of $2 \mathrm{~cm}^{-1} .{ }^{13}$ It was suggested the shape FLN spectra excited within the low energy $\mathrm{Q}_{\mathrm{y}}$-band were affected by an uncorrelated EET between pigments contributing to the two lowest-energy absorption bands. Below we use the analytical formulas (derived in this work for weakly coupled dimers), and apply them to describe the experimental FLN spectra reported in Ref. 35, which are re-plotted in Figure 6(b) for comparison. The relative intensities of ZPLs for experimental data are not available, as the spectra were measured with continuous mode $(\mathrm{CW})$ laser ${ }^{35}$ with a very narrow line width. In our modeling studies of the CP47 FLN spectra, in contrast to the calculations discussed above, we use the parameters from Ref. 13. That is, we assume that the site energies for Chl 523 and Chl 526 are $14420 \mathrm{~cm}^{-1}$ and $14562 \mathrm{~cm}^{-1}$, respectively. The FWHM of the corresponding Gaussian distributions are 150 and $190 \mathrm{~cm}^{-1}$, with the integrated band areas of 0.44 and 1.0, respectively. The inset in Figure 6(a) shows relative intensities of the calculated ZPLs that were cut off in the main frame where the shapes of PSBs were more clearly revealed.

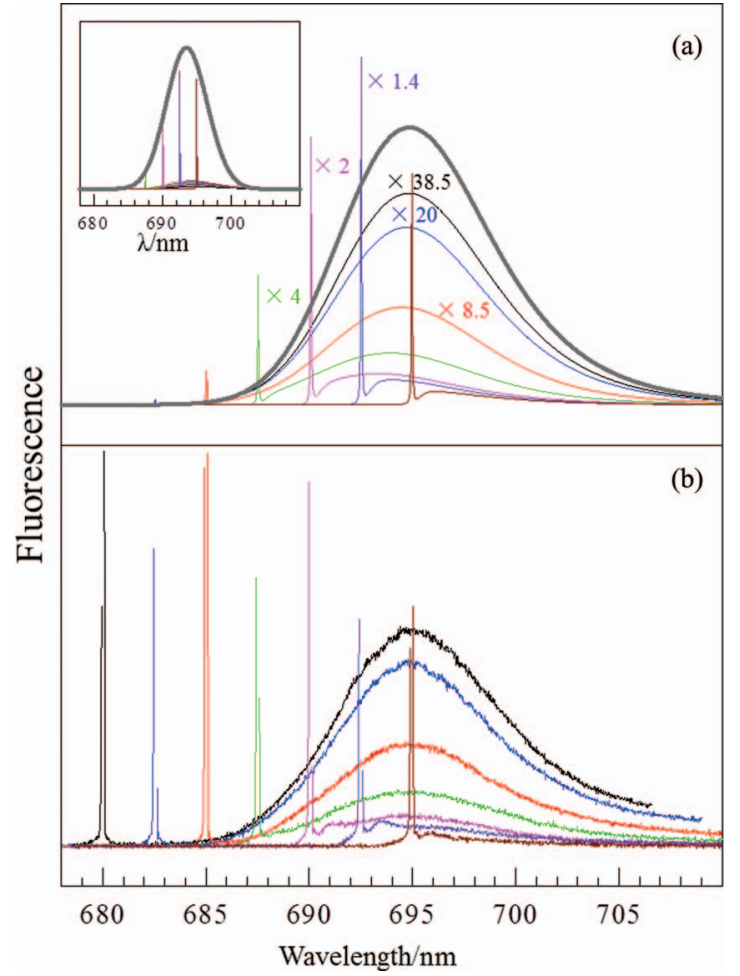

FIG. 6. Calculated (frame a) and experimental (frame b) FLN spectra of CP47. Analytical expression method is used for calculation, and only the two pigments with lowest site-energies are considered in the model (Chl 523 with site energy at $14420 \mathrm{~cm}^{-1}$ and $150 \mathrm{~cm}^{-1}$ FWHM; Chl 526 with site energy at $14562 \mathrm{~cm}^{-1}$ and $190 \mathrm{~cm}^{-1}$ FWHM). Mean phonon frequency is $26 \mathrm{~cm}^{-1}$. In each frame, black, blue, red, green, magenta, purple, and brown curves stand for excitation wavelengths at $680.0,682.5,685.0,687.5,690.0$, 692.5 , and $695.0 \mathrm{~nm}$, respectively. Thick gray line in frame (a) is the calculated bulk emission. Inset in frame (a) shows the relative intensities of ZPLs for calculated FLN spectra with respect to SDF of the lowest energy state.

Both calculated and experimental FLN spectra are qualitatively similar, that is, decreasing excitation wavelength (i.e., increasing excitation frequency) makes the shape of FLN spectra very similar to the non-resonantly excited emission (see the gray thick and black curves in frames (a) and (b) of Figure 6, respectively. The latter is caused by the uncorrelated EET from the second (higher-energy band) to the lowest energy trap from which emission occurs. Thus we conclude that the calculated FLN spectra for the CP47 complex using our analytical expressions developed in this work provide a good description of the experimental FLN spectra obtained for the CP47 antenna protein complex, and support our previous suggestion that some of the FLN spectra reported in Ref. 35 were affected by uncorrelated EET. This agreement further supports our earlier assignment that Chl 526 does not contribute solely to the lowest energy state since it is too weakly coupled to other pigments to produce either the low oscillator strength needed to fit the absorption and red-shifted emission spectra or the excitonic features of the persistent HB spectrum described in Ref. 13, in contrast to recent suggestions that $695 \mathrm{~nm}$ emission band observed in intact CP47 can be assigned to a single $\mathrm{Chl} 29^{24,36-39}$ (Chl numbering according to the nomenclature of Loll et al. ${ }^{34}$ ). 


\section{CONCLUSIONS}

This work describes simple analytical formulas for the absorption, emission, and fluorescence line-narrowed (FLN) spectra for weakly coupled pigments in the presence of uncorrelated EET between pigments contributing to the low energy absorption bands. The approach is demonstrated computationally for dimer systems, but is easily extended to describe FLN spectra for systems with more than two pigments, for example, antenna pigment complexes with multiple chromophores. We showed that in the weak el-ph coupling limit the derived formulas provide a good approximation to computationally expensive SCM simulations and, in particular, to the Redfield-approach calculated FLN spectra. Importantly, the analytical expressions provide significantly shorter computation time and smoother spectra than those obtained with the Redfield-type calculations. Application of the derived expressions to the FLN spectra obtained for the CP47 antenna protein complex ${ }^{35}$ (assuming an uncorrelated EET between two pigments contributing to the two nearly degenerate low-energy absorption bands) also yielded good qualitative agreement. Calculated and experimental FLN spectra for CP47 complex show very good qualitative agreement. Finally, our data do not support earlier Raszewski and Renger ${ }^{36}$ assignment that the red tail of the absorption spectrum of CP47 is localized on Chl 526 (or Chl 29 based on the numbering according to the nomenclature of Loll et al. ${ }^{40}$ ), which is responsible for the $695 \mathrm{~nm}$ emission. ${ }^{13}$ In contrast, the shapes of the FLN spectra, which clearly indicate the presence of uncorrelated EET between pigments contributing to the two lowest-energy (overlapping) are consistent with our assignment, ${ }^{13,35}$ i.e., the low-energy $\mathrm{Q}_{\mathrm{y}}$-absorption bands are mostly contributed to by two Chls, i.e., Chl523 (lowest energy state) and Chl526 (second lowest-energy state).

\section{ACKNOWLEDGMENTS}

This work was supported by the Chemical Sciences, Geosciences and Biosciences Division, Office of Basic Energy Sciences, Office of Science, U.S. Department of Energy (Grant No. DE-FG02-11ER16281 to R.J.). We acknowledge Adam Kell for help in computational part of this work and Dr. Valter Zazubovich for useful discussions.

${ }^{1}$ R. I. Personov, V. M. Agranovich, and R. M. Hochstrasser, Spectroscopy and Excitation Dynamic of Condensed Molecular Systems (North-Holland, Amsterdam, 1983), p. 555.

${ }^{2}$ R. Jankowiak and G. J. Small, Chem. Res. Toxicol. 4, 256 (1991).

${ }^{3}$ L. Nakhimovski, M. Lamotte, and J. Joussot-Dubien, Handbook of Low Temperature Electronic Spectra of Polycyclic Aromatic Hydrocarbons (Elsevier, Amsterdam/New York/Oxford/Tokyo, 1989).
${ }^{4}$ L. A. Riseberg, Phys. Rev. A 7, 671 (1973).

${ }^{5}$ F. Ariese, R. Jankowiak, C. Gooijer, and J. W. Hofstraat, Shpol'skii Spectroscopy and Other Site-Selection Methods (John Wiley and Sons, Inc., New York, 2000), p. 333.

${ }^{6}$ R. Jankowiak, M. Reppert, V. Zazubovich, J. Pieper, and T. Reinot, Chem. Rev. 111, 4546 (2011).

${ }^{7}$ A. Kell, X. Feng, M. Reppert, and R. Jankowiak, J. Phys. Chem. B 117, 7317 (2013).

${ }^{8}$ C. Lin, I. Renge, and R. Jankowiak, Chem. Phys. Lett. 576, 15 (2013).

${ }^{9}$ B. M. Kharlamov, R. I. Personov, and L. A. Bykovska, Opt. Commun. 12, 191 (1974).

${ }^{10}$ R. Purchase and S. Volker, Photosynth. Res. 101, 245 (2009).

${ }^{11}$ J. M. Hayes, J. K. Gillie, D. Tang, and G. J. Small, Biochim. Biophys. Acta 932, 287 (1988).

${ }^{12}$ R. Jankowiak, J. M. Hayes, and G. J. Small, Chem. Rev. 93, 1471 (1993).

${ }^{13}$ M. Reppert, K. Acharya, B. Neupane, and R. Jankowiak, J. Phys. Chem. B 114, 11884 (2010).

${ }^{14}$ T. Renger and R. A. Marcus, J. Chem. Phys. 116, 9997 (2002).

${ }^{15}$ K. Blum, Density Matrix Theory and Application (Plenum, New York, 1981).

${ }^{16}$ V. May and O. Kühn, Charge and Energy Transfer Dynamics in Molecular Systems: A Theoretical Introduction (Wiley-VCH, Berlin, 2000).

${ }^{17}$ O. Kühn, V. May, and M. Schreiber, J. Chem. Phys. 101, 10404 (1994).

${ }^{18}$ M. J. Reppert, Phys. Chem. Lett. 2, 2716 (2011).

${ }^{19}$ M. Schroder, U. Kleinekathofer, and M. Schreiber, J. Chem. Phys. 124, 084903 (2006)

${ }^{20}$ A. Ishizaki and G. R. Fleming, J. Chem. Phys. 130, 234110 (2009).

${ }^{21}$ V. I. Novoderezhkin and R. van Grondelle, Phys. Chem. Chem. Phys. 12, 7352 (2010).

${ }^{22}$ M. Reppert, V. Naibo, and R. Jankowiak, Chem. Phys. 367, 27 (2010).

${ }^{23}$ V. May and O. Kühn, Charge and Energy Transfer Dynamics in Molecular Systems, 2nd ed. (Wiley-VCH, Weinheim, 2004).

${ }^{24}$ Y. Shibata, S. Nishi, K. Kawakami, J.-R. Shen, and T. Renger, J. Am. Chem. Soc. 135, 6903 (2013).

${ }^{25}$ M. Wendling, T. Pullerits, M. A. Przyjalgowski, S. I. E. Vulto, T. J. Aartsma, R. van Grondelle, and H. J. van Amerongen, Phys. Chem. B 104, 5825 (2000).

${ }^{26}$ M. Reppert, V. Naibo, and R. Jankowiak, J. Chem. Phys. 133, 014506 (2010).

${ }^{27}$ J. M. Hayes, P. A. Lyle, and G. J. Small, J. Phys. Chem.-US 98, 7337 (1994).

${ }^{28}$ J. Pieper, J. Voigt, G. Renger, and G. J. Small, Chem. Phys. Lett. 310, 296 (1999).

${ }^{29}$ M. Rätsep, J. Pieper, K.-D. Irrgang, and A. Freiberg, J. Phys. Chem. B 112, 110 (2008).

${ }^{30}$ M. Rätsep and A. Freiberg, J. Lumin. 127, 251 (2007).

${ }^{31}$ T. Renger and V. May, Phys. Rev. Lett. 84, 5228 (2000).

${ }^{32}$ E. W. Knapp, Chem. Phys. 85, 73 (1984).

${ }^{33}$ A. Guskov, J. Kern, A. Gabdulkhakov, M. Broser, A. Zouni, and W. Saenger, Nat. Struct. Mol. Biol. 16, 334 (2009).

${ }^{34}$ B. Loll, J. Kern, W. Saenger, A. Zouni, and J. Biesiadka, Nature 438, 1040 (2005),.

${ }^{35}$ B. Neupane, N. C. Dang, K. Acharya, M. Reppert, V. Zazubovich, R. Picorel, M. Seibert, and R. Jankowiak, J. Am. Chem. Soc. 132, 4214 (2010).

${ }^{36}$ G. Raszewski and T. Renger, J. Am. Chem. Soc. 130, 4431 (2008).

${ }^{37}$ F. Mueh, T. Renger, and A. Zouni, Plant. Physiol. Bioch. 46, 238 (2008).

${ }^{38}$ T. Renger and E. Schlodder, J. Photoch. Photobio. B 104, 126 (2011).

${ }^{39}$ T. Renger and E. Schlodder, ChemPhysChem 11, 1141 (2010).

${ }^{40}$ B. Loll, J. Kern, A. Zouni, W. Saenger, J. Biesiadka, and K. D. Irrgang, Photosynth. Res. 86, 175 (2005).

${ }^{41}$ See supplementary material at http://dx.doi.org/10.1063/1.4887083 for additional examples of modeled FLN spectra in weakly coupled dimers obtained for different sets of parameters. 